\title{
CNC MACHINE RULED SURFACE INTERPOLATION: A NEURAL NETWORK APPROACH FOR CHEAP PROCESSING
}

\author{
Luis R. Canali, Mario R. Modesti, Eduardo A. Destéfanis \\ Grupo de Investigaciones en Informática para la Ingeniería \\ Universidad Tecnológica Nacional. Facultad Regional Córdoba \\ C.C. 36, Sucursal 16. 5016 Córdoba, Argentina \\ Fax: (+54)(351) 468 1823. E-mail: canali@sa.frc.utn.edu.ar
}

\begin{abstract}
Neural networks are an attractive approach to the problem of sculptured surface interpolation. They are very powerful because of their capability of performing over two-dimensional sets of data. Some discussion on NN interpolation capability is presented. Results of experiments with several curves are shown, and an outline of an environment suitable for a networked machine shop floor is introduced.
\end{abstract}

\section{INTRODUCTION}

Modern metalworking shop practice involves a lot of information going to and fro between the engineering and production environments within a plant. A good deal of this traffic is related to part programs involving the manufacture process of pieces designed around so-called sculptured surfaces. A growing number of cases appear daily in the auto-related industries, due to the use of these surfaces in dies of all kinds.

Sculptured surfaces do not have continuous mathematical definitions and they are usually result of styling rules. Their design cycle usually involves a model, from which measures are taken. These measures form a basic grid that is the starting point of the ruled surface, obtained by interpolation of these points.

There are a growing number of procedures for surface interpolation in the $\mathrm{CNC}$ environment. They are commonly related to some polynomial approach for grouping the mesh points in a patch-continuous mathematical form. A notable exception are the (Bézier 1972) and (Coons 1967) approximations. 
Polynomial methods of interpolation present uncomfortable oscillations associated with large number of data points: As the number of mesh points increase, so does the degree of the polynomial, causing lots of trouble when machining...

Neural networks have interesting mapping capabilities (Hagan, 1996; Orr, 1996, 1999), so interpolation is performed in a quite straightforward fashion. The paper discusses NN use, presenting some theoretical background on NN mapping. Comparison between surface interpolation performed with neural networks and spline functions is discussed, with some data regarding errors and processor time. The whole process asks for a networked factory environment because a lot of traffic is involved.

\section{2.- BACKGROUND}

Let $\mathbf{A}$ be a compact subset in $R^{N}$, and NN1 a backpropagating neural network. These networks have the capability of performing a good mapping of $\mathbf{A}$ into $\mathbf{f}(\mathbf{A})$ (Hagan, 1996; Bose, 1996; Orr, 1996), being this a closed subset of $\mathbf{A}$ in $R^{M}$.

$$
\mathrm{f}: / \mathbf{A} \in R^{N} \rightarrow \mathrm{f}[\mathbf{A}] \in R^{M}
$$

This is accomplished by "training" the network with examples $\left(\mathrm{x}_{1}, \mathrm{y}_{1}\right),\left(\mathrm{x}_{2}\right.$, $\left.y_{2}\right), \ldots\left(x_{k}, y_{k}\right)$, where

$$
\mathbf{y}_{\mathbf{k}}=\mathrm{f}\left(\mathbf{x}_{\mathbf{k}}\right)
$$

$y_{k}$ are system outputs and $x_{k}$ its inputs.

The quality of mapping can be very high. Let $\varepsilon$ be an arbitrarily small number. It can be shown that for some $\varepsilon>0$ and a square integrable, $L_{2}$ norm continuous function $\mathrm{f}$, such as:

$$
\mathrm{f}:[0,1]^{\mathrm{n}} \in R^{N} \rightarrow R^{M}
$$

there is a backpropagating $\mathrm{NN}$, with three layers, capable of approximate $\mathrm{f}$ within $\varepsilon$.

Proof of this can be done easily. $f$ can be expressed in terms of its vector components as:

$$
f(x)=\left(f_{1}(x), f_{2}(x), \ldots, f_{n}(x)\right)
$$

being $f_{1}(x)$ the l-th component of $f(x)$, it is possible to obtain

$$
\int_{[0,1]^{n}}\left|f_{1}(x)-\sum_{k} c_{l_{k}} e^{2 \pi j\left(k^{*} x\right)}\right|^{2} d x \pi \delta_{1}
$$

where $\left(k^{*} \mathbf{x}\right)$ denotes the vector product. The expression above can be expanded as a Fourier series, and each of the sine or cosine terms can be obtained by means of a three layer neural network. The resulting overall error, taking into account $n$ output elements is: 


$$
\int_{[0,1]^{\mathrm{n}}}\left|\mathrm{f}(\mathrm{x})-\mathrm{y}^{\prime}(\mathrm{x})\right|^{2} \mathrm{dx} \pi \varepsilon
$$

as was presented in the hypothesis.

This equation is very important. It states that $\mathrm{NN}$ can approximate, with relatively simple structures, continuous, square integrable functions with arbitrary nonzero errors. This constitutes no restriction. Almost every useful function is square integrable, at least patchwise, in $\mathrm{CNC}$ context.

\section{APPLICATIONS DISCUSSION}

\subsection{Spline and Neural Net approaches}

The current method for surface interpolation asks for a sequence of tasks that can be described as follows for a spline interpolation:

- Data (measurement) points of the surface are used for obtaining a basic (wide patch) grid.

- Using points from this grid, a second (much denser) grid is obtained. This grid is made by means of spline interpolation of sections of the surface along one of the coordinate system axes, and then completing the grid along the other axis.

Obviously, as splines cannot be used with two-dimensional data, this is a cumbersome and time-consuming task.

In the other hand, as the input space of a NN is n-dimensional (Hagan, 1996; Orr, 1996, 1999), the network can be trained using the measured points as described bellow:

- Network training is achieved by means of the measured points.

- A grid as dense as desired is obtained in a single operation using the trained network and an appropriately designed input vector.

To test the system two experiments were designed, both involving the kind of geometries common in CNC. The first used the set of tasks described for spline interpolation and produced a mesh using both spline and neural network interpolation of surface sections along one axis. Comparison was made with standard spline interpolation to study error behaviour.

The second experiment uses a bidimensional array of data for training a neural network and a bidimensional array for mesh generation. This experiment has no spline counterpart.

\subsection{Experiment one}

A framework for CNC applications requires errors within $.01 \mathrm{~mm}$ for surface interpolation. Several cases of both analytic (i.e. with known continuous mathematical definition) and empirical curves (defined by a set of measured points, and thereafter interpolated) were tested. Analytic curves used were trigonometric functions (sine, cosine and tangent), and quadratic and cubic parabolas. A piece of auto equipment was used as real world input to the system. Overall error was less than $3 \mathrm{E}-5 \mathrm{~mm}$ in all cases, and there were some specially interesting examples 
(curves representing trigonometric functions) for which maximum error was less than $1 \mathrm{E}-7 \mathrm{~mm}$. The following cases were studied:

- $y=\cos (x)$

- $y=\sin (x)$

- $y=\left(k^{2}-x^{2}\right)^{1 / 2}$ (circumference arc)

- $y=\tan (x)$

- $y=x^{3}+k$ (cubic parabola)

- $y=x^{2}+k$ (parabola)

- ruled curve.

For each case, a set of points with exact values were used for training the NN, and another close-spaced set of points as inputs for results check. In the experiment involving an auto piece, measurements were taken in a fashion standard throughout the industry: a coordinate measuring device was used attached to a high quality machine tool. Details are:

- Machine tool: Societé Genovoise d'Instruments de Phisique (GSIP) model MP$3 \mathrm{~K}$, serial $\mathrm{N}^{\circ}$ 9905, with an American TMS QA1 micrometer measuring system.

- $\quad$ TESA model QC300, serial $N^{\circ} 2$ FG 30023 measuring head.

- GSIP micrometer mounting gauges, used to construct an isostatic measuring environment.

A radial basis neural network, with three layers of neurons (one for input, a hidden layer and an output), was used. Experiments were performed using Matlab ${ }^{\circledR}$ packs for NN and spline functions. As these packages use the same IEEE arithmetic format, numeric consistency is ensured. Typical error behaviour is shown on fig. 1 to 8 for a representative section of the surface. Table 1 summarizes the error study.

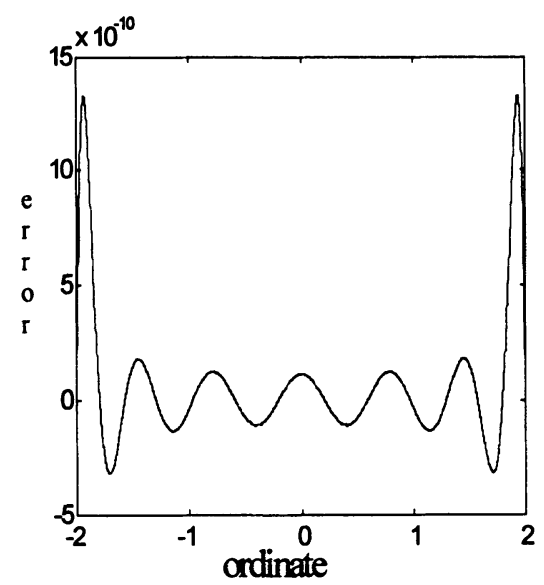

Figure 1. Error of the NN cosine function interpolation

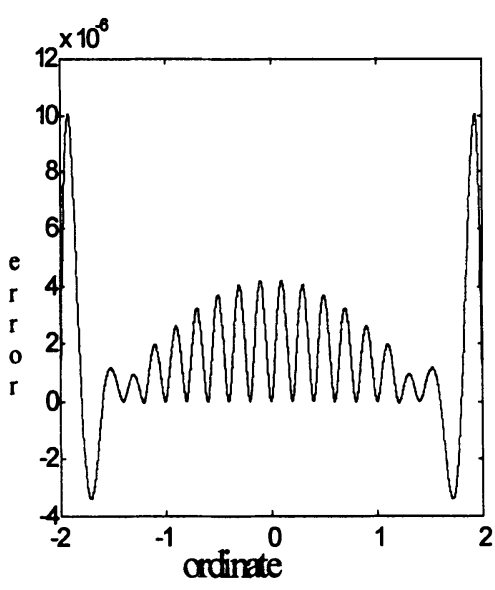

Figure 2. Error of the spline cosine function interpolation 


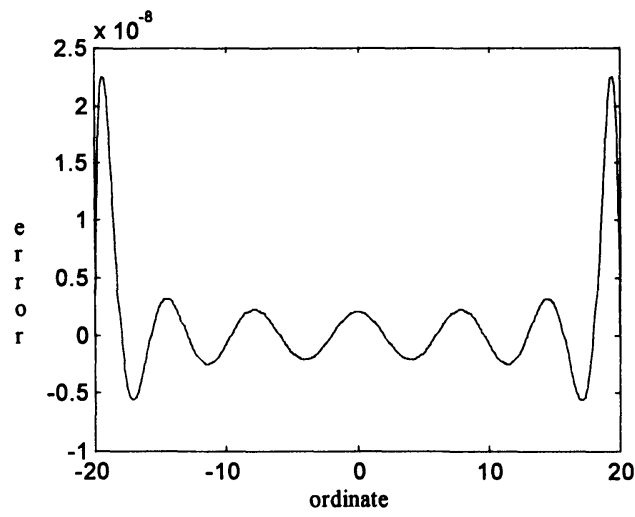

Figure 3. Error of NN arc of circumference interpolation

Fig. 4. Error of spline arc of circumference interpolation
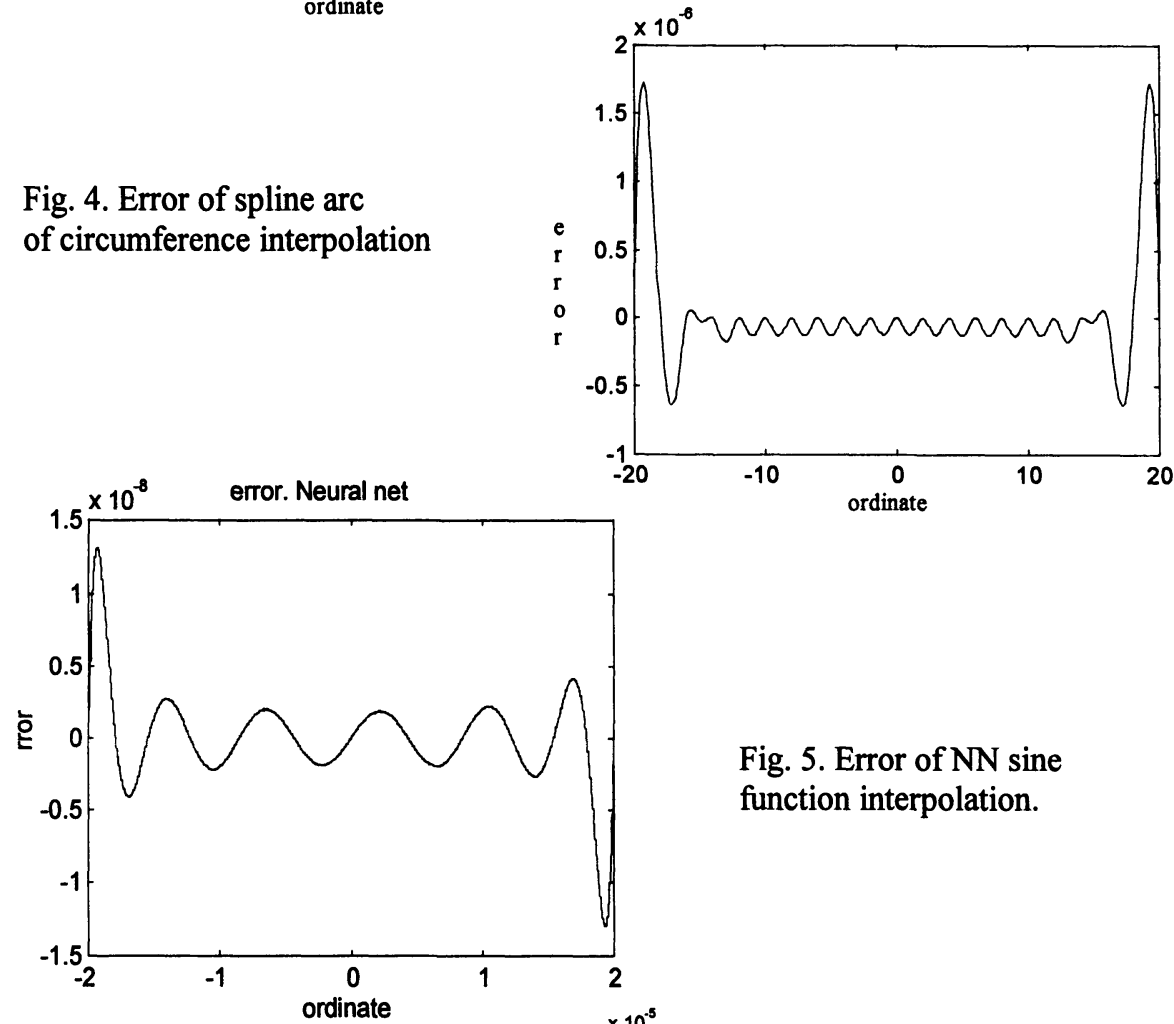

Fig. 6. Error of spline sine function interpolation.

Fig. 5. Error of NN sine function interpolation.

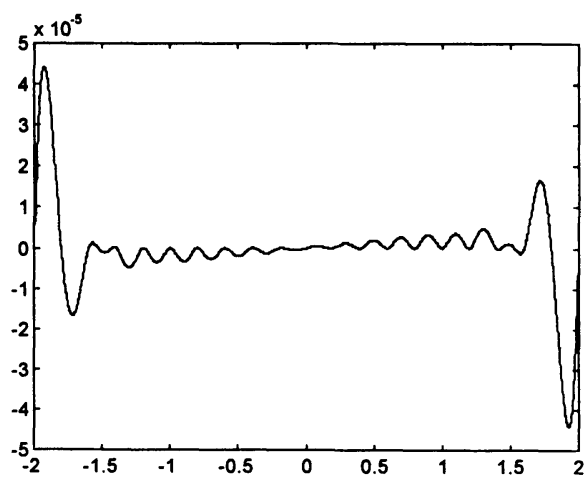




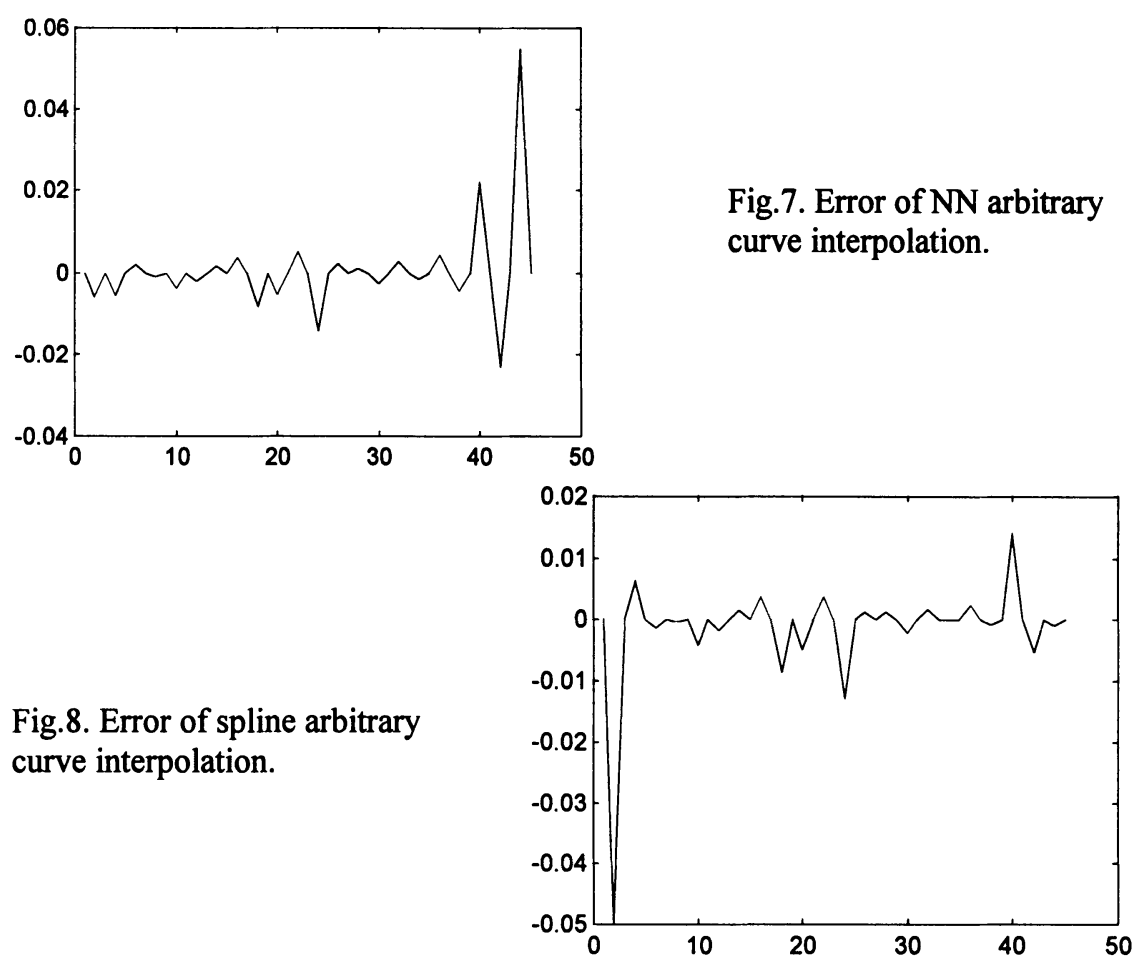

The following table summarizes errors encountered in several experiments.

Table 1. Maximum error values

\begin{tabular}{|l|ll|ll|}
\hline Function & error (NN) & $\begin{array}{l}\text { error } \\
\text { (splines) }\end{array}$ \\
\hline Cosine & 1.3322 & $\mathrm{E}-9$ & $1.0012 \mathrm{E}-5$ \\
\hline Parable & 3.6 & E-8 & 8.8818 & $\mathrm{E}-15$ \\
\hline Cubic Parable & 7.427 & $\mathrm{E}-6$ & 1.77 & $\mathrm{E}-15$ \\
\hline Arc of circumference & 2.256 & $\mathrm{E}-8$ & 1.7153 & $\mathrm{E}-6$ \\
\hline Sine & 1.3097 & $\mathrm{E}-8$ & 4.4064 & $\mathrm{E}-4$ \\
\hline Tangent & 7.09 & $\mathrm{E}-5$ & 4.97 & $\mathrm{E}-4$ \\
\hline
\end{tabular}

Measurements of computer time were made in each case using the built-in capabilities of the Matlab $\otimes$ package. The encountered values are shown in Table 2.

\subsection{Experiment two}

To test the algorithm, a patch of a sphere was used. For the sake of comfort it was centered at $z=-80$, with radius of 100 . To train the net, a series of equally spaced points was used as input vector. Point spacing was 1.0 and the test was performed in the interval $[1,10]$ for both $x$ and $y$.

Tests were performed with a non-homogeneous grid with .02 and .005 spacing in $\mathrm{x}$ and $\mathrm{y}$, respectively. The resulting error is shown in fig. 9. 
Table 2. Elapsed processor time for each case.

\begin{tabular}{|l|l|l|}
\hline \multicolumn{3}{|c|}{ Elapsed processor time [s] } \\
\hline \multicolumn{1}{|c|}{ function } & neural net & splines \\
\hline cosine & 2.41 & 1.81 \\
\hline parable & 1.93 & 0.82 \\
\hline cubic parable & 1.60 & 0.93 \\
\hline arc of circumference & 0.49 & 0.11 \\
\hline sine & 2.53 & 1.0 \\
\hline arbitrary curve (fourty points) & 0.44 & 0.99 \\
\hline tangent & 1.82 & 0.83 \\
\hline
\end{tabular}

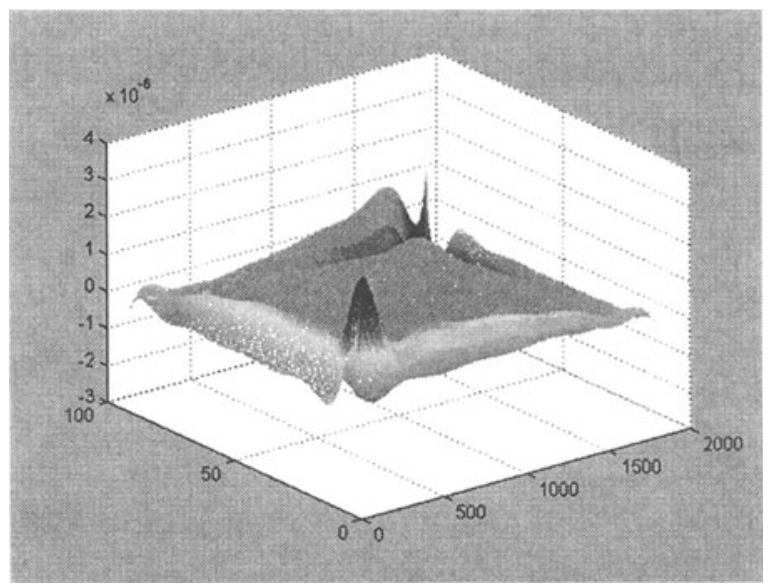

Figure 9. 3D plot of error (max. value 3.5 E-6)

\section{Discussion of error behaviour}

In experiment 1 errors were computed using a test grid of 4000 points for all cases, except for arc of circumference and arbitrary curve, where the number of points were 400 and 40 respectively. Tests were made using Matlab v 4.0 toolboxes running on a P5 PC running at $166 \mathrm{MHz}$ in a Windows NT environment.

Comparisons were made against spline interpolation of functions, because it is a well established industry procedure, used widely in the auto and die-cast shops.

The shape of error is relevant. Both types of approximation show similar features: Peak error occurs in the extremes of the interval and periodic behaviour is present. Peak error can be due to the fact that the algorithms used did not take into account the functions first derivative value at interval ends. The NN approach shows longer periods than splines with better peak values. The time needed for completing a whole mesh is considerable smaller using NNs, because of their two-dimensional interpolation capability. These properties make NN approximation of functions interesting from the shop floor point of view. It must be taken into account that all 
these procedures are made off-line, and do not interfere with machining. This approach lends itself to better finished pieces because of smoother error behaviour.

\section{IMPLEMENTATION.}

A set of programs was developed for an environment suitable for CNC shop. They are intended for all the process. They are meant to pick a series of measurements as input data and perform

- interpolation with splines

- interpolation using neural networks

- postprocessing of the geometric data to a generic DIN $66035 \mathrm{CNC}$, capable of machining straight lines and circular arcs.

The set is already running and suits superbly in a networked shop, where the final DIN 66025 language programs are relatively a minor part of overall traffic.

\section{ACKNOWLEDGEMENTS}

The authors wish to thank authorities of UTN for their support, and to personnel of Grupo de Investigaciones en Informática para la Ingeniería for their contributions to this work.

This work was carried on partially supported by grants from Consejo de Investigaciones de la Provincia de Córdoba (CONICOR) ( ${ }^{\circ}$ 4215/97) and from Agencia Nacional de Cooperación Científica y Tecnológica (ANCYT) No 11-0-358.

\section{REFERENCES:}

Bézier, P. "Numerical Control. Mathematics and Applications" J. Wiley \& Sons, London, 1972.

Bose, N. K.; Liang, P. "Neural Networks Fundamentals, with Graphs, Algorithms and Applications" McGraw-Hill, N. York, 1996

Coons, S. A. "Surfaces for computer-aided design of space forms". Technical Report MAC-TR-41. M.I.T., Mass. 1967.

Hagan, M. T.; Demuth, H. B.; Beale, M.

"Neural Network Design" PWS Publishing Company, Boston, 1996.

Orr, M. J. L. "Introduction to radial basis function networks" Technical report, Institute for Adaptive and Neural Computation. Edinburgh University, 1996. (www.anc.ed.ac.uk/mjo/papers/intro.ps)

Orr, M. J. L. "Matlab functions for radial basis function networks" Technical report, Institute for Adaptive and Neural Computation. Edinburgh University, 1999.

(www.anc.ed.ac.uk/mjo/software/rbf2.zip) 